\title{
SUPPLEMENTARY MATERIAL TO 'FLEXIBLE COVARIATE-ADJUSTED EXACT TESTS OF RANDOMIZED TREATMENT EFFECTS WITH APPLICATION TO A TRIAL OF HIV EDUCATION'
}

SUPPLEMENTARY MATERIAL

Supplement A: Small sample adjustment of Bickel (1978)

(http://lib.stat.cmu.edu/aoas/???/???). Function Definitions in Small sample approximation of $P(Z<z)$

For the class of statistics defined by $T=\sum_{i=1}^{n} A_{i} c_{i}$, where $c_{i}$ is a score, [? ] determined a higher-order approximation for the distribution of the standardized statistic $T^{*}$, given by

$$
\begin{aligned}
& P\left(T^{*}<t\right)= \\
& \Phi(t)-\frac{\phi(t)}{\pi(1-\pi)}\left[C_{1} H_{1}(t)+C_{2} H_{2}(t)+C_{3} H_{3}(t)+C_{5} H_{5}(t)\right], \text { where } \\
& C_{1}=\frac{\pi(1-\pi)}{2 n}, C_{2}=\frac{\sqrt{\pi(1-\pi)}(1-2 \pi)}{6} \frac{\sum_{i=1}^{n}(c-c .)^{3}}{\left\{\sum_{i=1}^{n}(c-c .)^{2}\right\}^{3 / 2}} \\
& C_{3}=\left\{\begin{array}{l}
\left.\frac{1-6 \pi+6 \pi^{2}}{24} \frac{\sum_{i=1}^{n}(c-c .)^{4}}{\left\{\sum_{i=1}^{n}(c-c .)^{2}\right\}^{2}}-\frac{(1-2 \pi)^{2}}{8 n}\right\}, \text { and } \\
C_{5}=\frac{(1-2 \pi)^{2}}{72} \frac{\left.\sum_{i=1}^{n}(c-c .)^{3}\right\}^{2}}{\left\{\sum_{i=1}^{n}(c-c .)^{2}\right\}^{3} .}
\end{array}\right.
\end{aligned}
$$

$H_{1}(t)-H_{5}(t)$ are given by the Hermite polynomials $H_{1}(t)=t, H_{2}(t)=$ $t^{2}-1, H_{3}(t)=t^{3}-3 t, H_{4}(t)=t^{4}-6 t^{2}+3$, and $H_{5}(t)=t^{5}-10 t^{3}+15 t$.

Supplement B: Simulation Study Tables - Independent Outcomes 
(http://lib.stat.cmu.edu/aoas/???/???). Type I error and power of covariateadjusted tests in independent outcomes. 
TABLE 1

Type I Error of Covariate-adjusted Tests. Conditional mean model (CMM), Augmented, Approx. Exact (without Bickel adjustment), Approx. Exact (BZ) (with Bickel adjustment) and Exact tests. Adaptive regression model selection: AIC, BIC, Adaptive LASSO (A. LASSO). Prespecified models: Correct, Incorrect. 'Unadjusted' denotes the test statistic that does not incorporate baseline covariates.

Conditional Mean Model

\begin{tabular}{|c|c|c|c|c|c|c|}
\hline$n_{a}$ & Unadjusted & $\mathrm{AIC}$ & $\mathrm{BIC}$ & A. LASSO & Incorrect & Correct \\
\hline 10 & 0.0394 & 0.209 & 0.1824 & 0.056 & 0.0354 & 0.0438 \\
\hline 15 & 0.0226 & 0.2554 & 0.1814 & 0.092 & 0.0352 & 0.045 \\
\hline 25 & 0.0288 & 0.1288 & 0.095 & 0.0576 & 0.0416 & 0.046 \\
\hline 50 & 0.0274 & 0.0758 & 0.0682 & 0.0546 & 0.0412 & 0.049 \\
\hline 100 & 0.0318 & 0.066 & 0.0592 & 0.054 & 0.0444 & 0.0452 \\
\hline \multicolumn{7}{|c|}{ Augmented } \\
\hline$n_{a}$ & Unadjusted & AIC & $\mathrm{BIC}$ & A. LASSO & Incorrect & Correct \\
\hline 10 & 0.0394 & 0.4254 & 0.3142 & 0.1162 & 0.1132 & 0.1224 \\
\hline 15 & 0.0226 & 0.4218 & 0.2646 & 0.1452 & 0.0768 & 0.092 \\
\hline 25 & 0.0288 & 0.1894 & 0.131 & 0.0836 & 0.061 & 0.0678 \\
\hline 50 & 0.0274 & 0.0978 & 0.0802 & 0.0644 & 0.052 & 0.0588 \\
\hline 100 & 0.0318 & 0.0758 & 0.0636 & 0.0582 & 0.0522 & 0.0526 \\
\hline
\end{tabular}

Approx. Exact

\begin{tabular}{ccccccc}
\hline$n_{a}$ & Unadjusted & AIC & BIC & A. LASSO & Incorrect & Correct \\
\hline 10 & 0.037 & 0.0378 & 0.0396 & 0.0384 & 0.0326 & 0.0394 \\
15 & 0.021 & 0.051 & 0.0492 & 0.0516 & 0.0336 & 0.0446 \\
25 & 0.0278 & 0.047 & 0.0454 & 0.0454 & 0.0404 & 0.0464 \\
50 & 0.027 & 0.0476 & 0.0496 & 0.0478 & 0.041 & 0.0492 \\
100 & 0.0314 & 0.0514 & 0.0526 & 0.0492 & 0.044 & 0.0452 \\
\hline
\end{tabular}

Approx. Exact (BZ)

\begin{tabular}{ccccccc}
\hline$n_{a}$ & Unadjusted & AIC & BIC & A. LASSO & Incorrect & Correct \\
\hline 10 & 0.0348 & 0.0366 & 0.037 & 0.0356 & 0.0302 & 0.0374 \\
15 & 0.022 & 0.049 & 0.0478 & 0.05 & 0.0338 & 0.0432 \\
25 & 0.0296 & 0.0462 & 0.0444 & 0.0448 & 0.0414 & 0.0464 \\
50 & 0.0308 & 0.048 & 0.0494 & 0.0482 & 0.043 & 0.0498 \\
100 & 0.0344 & 0.052 & 0.053 & 0.0498 & 0.045 & 0.0476 \\
\hline \multicolumn{7}{c}{} \\
& & Exact & & & \\
\hline$n_{a}$ & Unadjusted & AIC & BIC & A. LASSO & Incorrect & Correct \\
\hline 10 & 0.0482 & 0.046 & 0.0482 & 0.0454 & 0.0474 & 0.0476 \\
15 & 0.049 & 0.054 & 0.052 & 0.0542 & 0.048 & 0.0508 \\
25 & 0.0536 & 0.0488 & 0.0484 & 0.0486 & 0.0516 & 0.0512 \\
50 & 0.047 & 0.0496 & 0.0512 & 0.0494 & 0.0508 & 0.053 \\
100 & 0.0514 & 0.0542 & 0.0544 & 0.0512 & 0.0472 & 0.053 \\
\hline
\end{tabular}


TABLE 2

Power of Covariate-adjusted Tests when the correct model is a candidate model. Conditional mean model (CMM), Augmented, Approx. Exact (without Bickel adjustment), Approx. Exact (BZ) (with Bickel adjustment) and Exact tests. Adaptive regression model selection: AIC, BIC, Adaptive LASSO (A. LASSO). Prespecified models: Correct, Incorrect. 'Unadjusted' denotes the test statistic that does not incorporate baseline covariates.

Conditional Mean Model

\begin{tabular}{cccccccr}
\hline & $n_{a}$ & Unadjusted & AIC & BIC & A. LASSO & Incorrect & Correct \\
\hline 10 & 0.5538 & 0.856 & 0.8942 & 0.7934 & 0.831 & 0.7186 & \\
15 & 0.6812 & 0.9294 & 0.9412 & 0.8966 & 0.9126 & 0.8464 & \\
25 & 0.8286 & 0.9778 & 0.9784 & 0.9708 & 0.97 & 0.9488 & \\
50 & 0.9626 & 0.9948 & 0.9952 & 0.994 & 0.9944 & 0.9916 & \\
100 & 0.999 & 0.9996 & 0.9996 & 0.9996 & 0.9996 & 0.9996 & \\
\hline
\end{tabular}

Augmented

\begin{tabular}{cccccccr}
\hline & $n_{a}$ & Unadjusted & AIC & BIC & A. LASSO & Incorrect & Correct \\
\hline 10 & 0.5538 & 0.93 & 0.9256 & 0.8372 & 0.8902 & 0.8274 & \\
15 & 0.6812 & 0.9528 & 0.9542 & 0.9144 & 0.9336 & 0.895 & \\
25 & 0.8286 & 0.9828 & 0.9818 & 0.9746 & 0.974 & 0.9598 & \\
50 & 0.9626 & 0.9954 & 0.9954 & 0.9944 & 0.9942 & 0.9924 & \\
100 & 0.999 & 0.9996 & 0.9996 & 0.9996 & 0.9996 & 0.9996 & \\
\hline
\end{tabular}

Approx. Exact

\begin{tabular}{cccccccr}
\multicolumn{7}{c}{ Approx. Exact } \\
\hline & $n_{a}$ & Unadjusted & AIC & BIC & A. LASSO & Incorrect & Correct \\
\hline 10 & 0.5444 & 0.509 & 0.6506 & 0.7048 & 0.8064 & 0.686 & \\
15 & 0.676 & 0.6606 & 0.8342 & 0.8554 & 0.9078 & 0.8358 & \\
25 & 0.8274 & 0.9522 & 0.9662 & 0.9626 & 0.9698 & 0.9466 & \\
50 & 0.9622 & 0.994 & 0.9946 & 0.9936 & 0.9942 & 0.9914 & \\
100 & 0.999 & 0.9996 & 0.9996 & 0.9996 & 0.9996 & 0.9996 & \\
\hline
\end{tabular}

Approx. Exact (BZ)

\begin{tabular}{cccccccr}
\hline & $n_{a}$ & Unadjusted & AIC & BIC & A. LASSO & Incorrect & Correct \\
\hline 10 & 0.5364 & 0.5012 & 0.64 & 0.697 & 0.8014 & 0.676 & \\
15 & 0.675 & 0.6554 & 0.831 & 0.8538 & 0.9082 & 0.8354 & \\
25 & 0.828 & 0.9528 & 0.967 & 0.9636 & 0.971 & 0.9466 & \\
50 & 0.963 & 0.994 & 0.995 & 0.994 & 0.9946 & 0.9922 & \\
100 & 0.999 & 0.9996 & 0.9996 & 0.9996 & 0.9996 & 0.9996 & \\
\hline
\end{tabular}

\begin{tabular}{cccccccr}
\multicolumn{7}{c}{ Exact } \\
\hline & $n_{a}$ & Unadjusted & AIC & BIC & A. LASSO & Incorrect & Correct \\
\hline 10 & 0.5784 & 0.5292 & 0.6682 & 0.721 & 0.8226 & 0.7054 & \\
15 & 0.6948 & 0.669 & 0.8398 & 0.864 & 0.918 & 0.8464 & \\
25 & 0.8382 & 0.9578 & 0.9692 & 0.9674 & 0.9744 & 0.952 & \\
50 & 0.966 & 0.9952 & 0.9958 & 0.9954 & 0.9962 & 0.993 & \\
100 & 0.999 & 0.9998 & 0.9998 & 0.9998 & 0.9998 & 0.9998 & \\
\hline
\end{tabular}


TABLE 3

Power of Covariate-adjusted Tests when the correct model is not a candidate model. Conditional mean model (CMM), Augmented, Approx. Exact (without Bickel adjustment), Approx. Exact (BZ) (with Bickel adjustment) and Exact tests. Adaptive regression model selection: AIC, BIC, Adaptive LASSO (A. LASSO). Prespecified models: Correct, Incorrect. 'Unadjusted' denotes the test statistic that does not incorporate baseline covariates.

Conditional Mean Model

\begin{tabular}{ccccccc}
\hline$n_{a}$ & Unadjusted & AIC & BIC & A. LASSO & Incorrect & Correct \\
\hline 10 & 0.1348 & 0.5982 & 0.638 & 0.503 & 0.8324 & 0.3496 \\
15 & 0.1498 & 0.6606 & 0.6604 & 0.5528 & 0.9118 & 0.3978 \\
25 & 0.1542 & 0.6598 & 0.6494 & 0.5892 & 0.97 & 0.4576 \\
50 & 0.1874 & 0.6792 & 0.6696 & 0.6404 & 0.9942 & 0.5454 \\
100 & 0.2398 & 0.7116 & 0.7066 & 0.6868 & 0.9996 & 0.6154 \\
\hline \multicolumn{7}{c}{ Augmented } \\
\hline$n_{a}$ & Unadjusted & AIC & BIC & A. LASSO & Incorrect & Correct \\
\hline 10 & 0.1348 & 0.7852 & 0.7448 & 0.6018 & 0.6932 & 0.4948 \\
15 & 0.1498 & 0.771 & 0.7196 & 0.6258 & 0.7166 & 0.49 \\
25 & 0.1542 & 0.714 & 0.6856 & 0.63 & 0.763 & 0.5108 \\
50 & 0.1874 & 0.7042 & 0.6878 & 0.6614 & 0.8252 & 0.5674 \\
100 & 0.2398 & 0.721 & 0.7136 & 0.695 & 0.8794 & 0.6256 \\
\hline \multicolumn{7}{c}{} \\
\hline$n_{a}$ & Unadjusted & AIC & Approx. Exact & & \\
\hline 10 & 0.1278 & 0.2516 & 0.3276 & 0.3748 & 0.8132 & 0.328 \\
15 & 0.1456 & 0.3162 & 0.423 & 0.4592 & 0.9076 & 0.388 \\
25 & 0.151 & 0.511 & 0.5432 & 0.552 & 0.97 & 0.4516 \\
50 & 0.186 & 0.6174 & 0.6284 & 0.623 & 0.9944 & 0.543 \\
100 & 0.2396 & 0.6872 & 0.686 & 0.6802 & 0.9994 & 0.6154 \\
\hline
\end{tabular}

Approx. Exact (BZ)

\begin{tabular}{ccccccc}
\hline$n_{a}$ & Unadjusted & AIC & BIC & A. LASSO & Incorrect & Correct \\
\hline 10 & 0.1268 & 0.242 & 0.3172 & 0.3682 & 0.809 & 0.3206 \\
15 & 0.1482 & 0.3106 & 0.4184 & 0.4546 & 0.9076 & 0.386 \\
25 & 0.1576 & 0.5078 & 0.5406 & 0.5488 & 0.9714 & 0.4516 \\
50 & 0.194 & 0.6174 & 0.6276 & 0.6232 & 0.9948 & 0.545 \\
100 & 0.2478 & 0.6892 & 0.6874 & 0.6814 & 0.9996 & 0.6192 \\
\hline
\end{tabular}

Exact

\begin{tabular}{ccccccc}
\hline$n_{a}$ & Unadjusted & AIC & BIC & A. LASSO & Incorrect & Correct \\
\hline 10 & 0.2178 & 0.269 & 0.3434 & 0.3884 & 0.8306 & 0.3452 \\
15 & 0.221 & 0.3218 & 0.4316 & 0.467 & 0.9182 & 0.4012 \\
25 & 0.2204 & 0.5152 & 0.5484 & 0.5556 & 0.976 & 0.4642 \\
50 & 0.244 & 0.6228 & 0.6332 & 0.6278 & 0.996 & 0.5542 \\
100 & 0.2942 & 0.6892 & 0.6908 & 0.6844 & 0.9998 & 0.631 \\
\hline
\end{tabular}


Supplement C: Simulation Study Tables - Dependent Outcomes

(http://lib.stat.cmu.edu/aoas/???/???). Type I Error under low correlation and power under low correlation and high correlation of covariate-adjusted tests for clustered outcomes

TABLE 4

Type I Error of CMM and Augmented Tests: low correlation, modeled after Young Citizens. Rows 1-3 contain results for cluster size $m_{i}=(20,30)$. Rows 4-6 show results for $m_{i}=(4,6,8)$. Adaptive regression model selection: AIC, BIC by $n$ (BICn), BIC by M,(BICm), Adaptive LASSO (A. L.). Prespecified models: Correct (Corr.), Incorrect (Inco.). 'Unadj.' denotes the test statistic that does not incorporate baseline covariates.

\begin{tabular}{lllllllll} 
& \multicolumn{7}{c}{ Conditional Mean Model } \\
\hline Large $m_{i}$ & $n_{a}$ & Unadjusted & AIC & BICn & BICm & A. LASSO & Correct & Incorrect \\
& 15 & 0.0673 & 0.2096 & 0.1799 & 0.1459 & 0.1417 & 0.0872 & 0.0826 \\
& 25 & 0.0620 & 0.1290 & 0.1143 & 0.0930 & 0.1027 & 0.0603 & 0.0623 \\
\hline Small $m_{i}$ & 25 & 0.0567 & 0.0843 & 0.0770 & 0.0707 & 0.0757 & 0.0587 & 0.0610 \\
\hline & 50 & 0.0443 & 0.0870 & 0.0760 & 0.0687 & 0.0643 & 0.0547 & 0.0553 \\
& 100 & 0.0484 & 0.0563 & 0.0523 & 0.0503 & 0.0487 & 0.0460 & 0.0463 \\
\hline
\end{tabular}

\begin{tabular}{lllllllll} 
& \multicolumn{8}{c}{ Augmented } \\
\hline & $n_{a}$ & Unadjusted & AIC & BICn & BICm & A. LASSO & Correct & Incorrect \\
\hline Large $m_{i}$ & 10 & 0.0673 & 0.2530 & 0.2148 & 0.1619 & 0.1760 & 0.1152 & 0.1133 \\
& 15 & 0.0620 & 0.1540 & 0.1277 & 0.1023 & 0.1327 & 0.0797 & 0.0747 \\
& 25 & 0.0593 & 0.1013 & 0.0873 & 0.0800 & 0.0970 & 0.0677 & 0.0713 \\
\hline Smallm $_{i}$ & 25 & 0.0567 & 0.1050 & 0.0830 & 0.0717 & 0.0760 & 0.0670 & 0.0690 \\
& 50 & 0.0443 & 0.0637 & 0.0550 & 0.0533 & 0.0533 & 0.0507 & 0.0497 \\
& 100 & 0.0484 & 0.0500 & 0.0480 & 0.0465 & 0.0500 & 0.0461 & 0.0539 \\
\hline
\end{tabular}


TABLE 5

Type I Error of Approximate Exact Tests: low correlation, modeled after Young Citizens. Rows 1-3 contain results for cluster size $m_{i}=(20,30)$. Rows 4-6 show results for $m_{i}=(4,6,8)$. Adaptive regression model selection: AIC, BIC by $n$ (BICn), BIC by M,(BICm), Adaptive LASSO (A. L.). Prespecified models: Correct (Corr.), Incorrect (Inco.). 'Unadj.' denotes the test statistic that does not incorporate baseline covariates.

Approximate Exact (Ind)

\begin{tabular}{lllllllll}
\hline & $n_{a}$ & Unadjusted & AIC & BICn & BICm & A. LASSO & Correct & Incorrect \\
\hline Large $m_{i}$ & 10 & 0.0349 & 0.0346 & 0.0379 & 0.0421 & 0.0349 & 0.0320 & 0.0349 \\
& 15 & 0.0423 & 0.0450 & 0.0453 & 0.0407 & 0.0423 & 0.0340 & 0.0343 \\
& 25 & 0.0453 & 0.0480 & 0.0450 & 0.0457 & 0.0470 & 0.0410 & 0.0407 \\
\hline Small $m_{i}$ & 25 & 0.0413 & 0.0410 & 0.0390 & 0.0400 & 0.0403 & 0.0390 & 0.0397 \\
& 50 & 0.0387 & 0.0377 & 0.0383 & 0.0393 & 0.0393 & 0.0377 & 0.0370 \\
& 100 & 0.0437 & 0.0387 & 0.0406 & 0.0398 & 0.0402 & 0.0371 & 0.0441 \\
\hline
\end{tabular}

Approximate Exact (Ind-Sm)

\begin{tabular}{lllllllll} 
& \multicolumn{8}{c}{ Approximate Exact (Ind-Sm) } \\
\hline & $n_{a}$ & Unadjusted & AIC & BICn & BICm & A. LASSO & Correct & Incorrect \\
\hline Large $m_{i}$ & 10 & 0.0379 & 0.0372 & 0.0411 & 0.0447 & 0.0379 & 0.0353 & 0.0385 \\
& 15 & 0.0433 & 0.0470 & 0.0473 & 0.0427 & 0.0430 & 0.0357 & 0.0377 \\
& 25 & 0.0477 & 0.0513 & 0.0487 & 0.0483 & 0.0497 & 0.0427 & 0.0430 \\
\hline Small $m_{i}$ & 25 & 0.0437 & 0.0433 & 0.0420 & 0.0427 & 0.0453 & 0.0403 & 0.0417 \\
& 50 & 0.0403 & 0.0393 & 0.0413 & 0.0413 & 0.0420 & 0.0400 & 0.0393 \\
& 100 & 0.0453 & 0.0402 & 0.0433 & 0.0418 & 0.0433 & 0.0390 & 0.0457 \\
\hline
\end{tabular}

\begin{tabular}{lllllllll}
\hline & \multicolumn{8}{c}{ Approximate Exact (Exch) } \\
\hline Large $m_{i}$ & $n_{a}$ & Unadjusted & AIC & BICn & BICm & A. LASSO & Correct & Incorrect \\
& 15 & 0.0356 & 0.0330 & 0.0369 & 0.0411 & 0.0333 & 0.0320 & 0.0346 \\
& 25 & 0.0403 & 0.0447 & 0.0453 & 0.0403 & 0.0430 & 0.0347 & 0.0363 \\
\hline Small $m_{i}$ & 25 & 0.0407 & 0.0490 & 0.0490 & 0.0493 & 0.0480 & 0.0413 & 0.0417 \\
& 50 & 0.0367 & 0.0403 & 0.0430 & 0.0413 & 0.0420 & 0.0393 & 0.0387 \\
& 100 & 0.0422 & 0.0375 & 0.0377 & 0.0373 & 0.0363 & 0.0390 & 0.0383 \\
\end{tabular}

Approximate Exact (Exch-Sm)

\begin{tabular}{lllllllll}
\hline & $n_{a}$ & Unadjusted & AIC & BICn & BICm & A. LASSO & Correct & Incorrect \\
\hline Large $m_{i}$ & 10 & 0.0366 & 0.0356 & 0.0395 & 0.0447 & 0.0359 & 0.0356 & 0.0379 \\
& 15 & 0.0423 & 0.0470 & 0.0470 & 0.0423 & 0.0450 & 0.0383 & 0.0397 \\
& 25 & 0.0473 & 0.0507 & 0.0510 & 0.0510 & 0.0510 & 0.0430 & 0.0440 \\
\hline Small $m_{i}$ & 25 & 0.0440 & 0.0437 & 0.0453 & 0.0433 & 0.0453 & 0.0417 & 0.0413 \\
& 50 & 0.0390 & 0.0387 & 0.0407 & 0.0403 & 0.0383 & 0.0407 & 0.0410 \\
& 100 & 0.0445 & 0.0398 & 0.0410 & 0.0414 & 0.0433 & 0.0394 & 0.0469 \\
\hline
\end{tabular}


TABLE 6

Type I Error of Exact Tests: low correlation, modeled after Young Citizens. Rows 1-3 contain results for cluster size $m_{i}=(20,30)$. Rows $4-6$ show results for $m_{i}=(4,6,8)$. Adaptive regression model selection: $A I C, B I C$ by $n$ (BICn), BIC by M,(BICm), Adaptive LASSO (A. L.). Prespecified models: Correct (Corr.), Incorrect (Inco.). 'Unadj.' denotes the test statistic that does not incorporate baseline covariates.

\begin{tabular}{lllllllll} 
& \multicolumn{7}{c}{ Exact (Ind) } \\
\hline Large $m_{i}$ & $n_{a}$ & Unadjusted & AIC & BICn & BICm & A. LASSO & Correct & Incorrect \\
& 15 & 0.0496 & 0.0434 & 0.0483 & 0.0506 & 0.0441 & 0.0457 & 0.0493 \\
& 25 & 0.0533 & 0.0540 & 0.0533 & 0.0500 & 0.0467 & 0.0480 & 0.0430 \\
\hline Small $m_{i}$ & 25 & 0.0483 & 0.0547 & 0.0543 & 0.0540 & 0.0547 & 0.0500 & 0.0513 \\
& 50 & 0.0413 & 0.0530 & 0.0487 & 0.0503 & 0.0533 & 0.0517 & 0.0480 \\
& 100 & 0.0469 & 0.0413 & 0.0440 & 0.0450 & 0.0443 & 0.0437 & 0.0447 \\
\end{tabular}

\begin{tabular}{lllllllll} 
& \multicolumn{7}{c}{ Exact (Exch) } \\
\hline Large $m_{i}$ & $n_{a}$ & Unadjusted & AIC & BICn & BICm & A. LASSO & Correct & Incorrect \\
& 15 & 0.0457 & 0.0421 & 0.0480 & 0.0503 & 0.0424 & 0.0470 & 0.0473 \\
& 25 & 0.0523 & 0.0540 & 0.0530 & 0.0497 & 0.0480 & 0.0503 & 0.0443 \\
Small $m_{i}$ & 25 & 0.0510 & 0.0577 & 0.0580 & 0.0553 & 0.0570 & 0.0497 & 0.0513 \\
& 50 & 0.0430 & 0.0513 & 0.0523 & 0.0517 & 0.0543 & 0.0523 & 0.0510 \\
& 100 & 0.0461 & 0.0407 & 0.0427 & 0.0433 & 0.0427 & 0.0450 & 0.0447 \\
\end{tabular}

TABLE 7

Power of CMM and Augmented Tests: low correlation, modeled after Young Citizens. Rows 1-3 contain results for cluster size $m_{i}=(20,30)$. Rows 4- 6 show results for $m_{i}=(4,6,8)$. Adaptive regression model selection: $A I C, B I C$ by $n$ (BICn), BIC by M,(BICm), Adaptive LASSO (A. L.). Prespecified models: Correct (Corr.), Incorrect (Inco.). 'Unadj.' denotes the test statistic that does not incorporate baseline covariates.

\begin{tabular}{lllllllll}
\multicolumn{7}{c}{ Conditional Mean Model } \\
\hline & $n_{a}$ & Unadjusted & AIC & BICn & BICm & A. LASSO & Correct & Incorrect \\
\hline Small $m_{i}$ & 10 & 0.2347 & 0.4646 & 0.4486 & 0.4238 & 0.3970 & 0.3666 & 0.3271 \\
& 15 & 0.2960 & 0.4829 & 0.4735 & 0.4594 & 0.4523 & 0.4312 & 0.4019 \\
& 25 & 0.3572 & 0.5513 & 0.5479 & 0.5355 & 0.5310 & 0.5212 & 0.4851 \\
\hline Large $m_{i}$ & 25 & 0.2557 & 0.3860 & 0.3680 & 0.3583 & 0.3493 & 0.3369 & 0.3164 \\
& 50 & 0.3890 & 0.4964 & 0.4912 & 0.4870 & 0.4820 & 0.4796 & 0.4548 \\
& 100 & 0.5670 & 0.6713 & 0.6667 & 0.6640 & 0.6623 & 0.6573 & 0.6350 \\
\hline \multirow{2}{*}{ Small $m_{i}$} & 10 & 0.2347 & 0.5100 & 0.4822 & 0.4339 & 0.4391 & 0.4071 & 0.3617 \\
& 15 & 0.2960 & 0.5059 & 0.4887 & 0.4694 & 0.4809 & 0.4607 & 0.4288 \\
& 25 & 0.3572 & 0.5656 & 0.5550 & 0.5429 & 0.5505 & 0.5383 & 0.5026 \\
\hline Large $m_{i}$ & 25 & 0.2557 & 0.3963 & 0.3752 & 0.3601 & 0.3541 & 0.3572 & 0.3322 \\
& 50 & 0.3890 & 0.4984 & 0.4872 & 0.4848 & 0.4830 & 0.4876 & 0.4670 \\
& 100 & 0.5670 & 0.6700 & 0.6673 & 0.6640 & 0.6590 & 0.6613 & 0.6430 \\
\hline
\end{tabular}


TABLE 8

Power of Approximate Exact Tests: low correlation, modeled after Young Citizens.

Rows 1-3 contain results for cluster size $m_{i}=(20,30)$. Rows 4-6 show results for $m_{i}=(4,6,8)$. Adaptive regression model selection: AIC, BIC by n (BICn), BIC by M,(BICm), Adaptive LASSO (A. L.). Prespecified models: Correct (Corr.), Incorrect (Inco.). 'Unadj.' denotes the test statistic that does not incorporate baseline covariates.

Approximate Exact (Ind)

\begin{tabular}{lllllllll}
\hline & $n_{a}$ & Unadjusted & AIC & BICn & BICm & A. LASSO & Correct & Incorrect \\
\hline Small $m_{i}$ & 10 & 0.1303 & 0.1649 & 0.1815 & 0.2145 & 0.1740 & 0.2295 & 0.2005 \\
& 15 & 0.1953 & 0.2962 & 0.3136 & 0.3303 & 0.2969 & 0.3379 & 0.3114 \\
& 25 & 0.2678 & 0.4372 & 0.4580 & 0.4653 & 0.4289 & 0.4686 & 0.4352 \\
\hline Large $m_{i}$ & 25 & 0.1743 & 0.2658 & 0.2847 & 0.2853 & 0.2655 & 0.2871 & 0.2650 \\
& 50 & 0.2938 & 0.4278 & 0.4422 & 0.4410 & 0.4360 & 0.4460 & 0.4194 \\
& 100 & 0.4650 & 0.6420 & 0.6460 & 0.6423 & 0.6380 & 0.6457 & 0.6230 \\
\hline
\end{tabular}

Approximate Exact (Ind-Sm)

\begin{tabular}{lllllllll}
\hline & $n_{a}$ & Unadjusted & AIC & BICn & BICm & A. LASSO & Correct & Incorrect \\
\hline Small $m_{i}$ & 10 & 0.1368 & 0.1701 & 0.1874 & 0.2204 & 0.1815 & 0.2364 & 0.2086 \\
& 15 & 0.2014 & 0.3034 & 0.3214 & 0.3383 & 0.3040 & 0.3479 & 0.3186 \\
& 25 & 0.2730 & 0.4448 & 0.4625 & 0.4703 & 0.4354 & 0.4736 & 0.4413 \\
\hline Large $m_{i}$ & 25 & 0.1793 & 0.2739 & 0.2905 & 0.2921 & 0.2745 & 0.2921 & 0.2734 \\
& 50 & 0.3008 & 0.4364 & 0.4486 & 0.4492 & 0.4436 & 0.4542 & 0.4266 \\
& 100 & 0.4743 & 0.6477 & 0.6530 & 0.6500 & 0.6463 & 0.6513 & 0.6300 \\
\hline
\end{tabular}

\begin{tabular}{lllllllll} 
& \multicolumn{8}{c}{ Approximate Exact (Exch) } \\
\hline & $n_{a}$ & Unadjusted & AIC & BICn & BICm & A. LASSO & Correct & Incorrect \\
\hline Small $m_{i}$ & 10 & 0.1587 & 0.1639 & 0.1815 & 0.2138 & 0.1756 & 0.2298 & 0.2031 \\
& 15 & 0.2363 & 0.2941 & 0.3134 & 0.3301 & 0.2984 & 0.3372 & 0.3092 \\
& 25 & 0.3219 & 0.4350 & 0.4571 & 0.4647 & 0.4328 & 0.4677 & 0.4348 \\
\hline Large $m_{i}$ & 25 & 0.2027 & 0.2700 & 0.2861 & 0.2887 & 0.2665 & 0.2897 & 0.2676 \\
& 50 & 0.3428 & 0.4334 & 0.4432 & 0.4432 & 0.4372 & 0.4490 & 0.4244 \\
& 100 & 0.5263 & 0.6387 & 0.6480 & 0.6487 & 0.6427 & 0.6480 & 0.6257 \\
\hline
\end{tabular}

Approximate Exact (Exch-Sm)

\begin{tabular}{lllllllll}
\hline & $n_{a}$ & Unadjusted & AIC & BICn & BICm & A. LASSO & Correct & Incorrect \\
\hline Small $m_{i}$ & 10 & 0.1632 & 0.1704 & 0.1877 & 0.2227 & 0.1818 & 0.2338 & 0.2086 \\
& 15 & 0.2422 & 0.3021 & 0.3227 & 0.3388 & 0.3056 & 0.3464 & 0.3164 \\
& 25 & 0.3276 & 0.4415 & 0.4647 & 0.4723 & 0.4406 & 0.4751 & 0.4396 \\
\hline Large $m_{i}$ & 25 & 0.2072 & 0.2787 & 0.2940 & 0.2966 & 0.2739 & 0.2950 & 0.2752 \\
& 50 & 0.3490 & 0.4408 & 0.4508 & 0.4504 & 0.4444 & 0.4588 & 0.4340 \\
& 100 & 0.5333 & 0.6450 & 0.6543 & 0.6547 & 0.6490 & 0.6523 & 0.6330 \\
\hline
\end{tabular}


TABLE 9

Power of Exact Tests: low correlation, modeled after Young Citizens. Rows 1-3 contain results for cluster size $m_{i}=(20,30)$. Rows $4-6$ show results for $m_{i}=(4,6,8)$. Adaptive regression model selection: $A I C, B I C$ by $n$ (BICn), BIC by $M,(B I C m)$,

Adaptive LASSO (A. L.). Prespecified models: Correct (Corr.), Incorrect (Inco.).

'Unadj.' denotes the test statistic that does not incorporate baseline covariates.

\begin{tabular}{|c|c|c|c|c|c|c|c|c|}
\hline \multicolumn{9}{|c|}{ Exact (Ind) } \\
\hline & $n_{a}$ & Unadjusted & AIC & BICn & BICm & A. LASSO & Correct & Incorrect \\
\hline \multirow{3}{*}{ Small $m_{i}$} & 10 & 0.1534 & 0.1864 & 0.2047 & 0.2341 & 0.1943 & 0.2618 & 0.2344 \\
\hline & 15 & 0.2122 & 0.3171 & 0.3353 & 0.3531 & 0.3190 & 0.3707 & 0.3411 \\
\hline & 25 & 0.2838 & 0.4584 & 0.4738 & 0.4827 & 0.4513 & 0.4909 & 0.4549 \\
\hline \multirow[t]{3}{*}{ Large $m_{i}$} & 25 & 0.1901 & 0.2845 & 0.3027 & 0.3048 & 0.2871 & 0.3156 & 0.2892 \\
\hline & 50 & 0.3064 & 0.4506 & 0.4596 & 0.4592 & 0.4552 & 0.4682 & 0.4420 \\
\hline & 100 & 0.4810 & 0.6590 & 0.6630 & 0.6610 & 0.6563 & 0.6613 & 0.6377 \\
\hline \multicolumn{9}{|c|}{ Exact (Exch) } \\
\hline & $n_{a}$ & Unadjusted & $\mathrm{AIC}$ & $\mathrm{BICn}$ & $\mathrm{BICm}$ & A. LASSO & Correct & Incorrect \\
\hline \multirow[t]{3}{*}{ Small $m_{i}$} & 10 & 0.1867 & 0.1851 & 0.2027 & 0.2344 & 0.1975 & 0.2618 & 0.2308 \\
\hline & 15 & 0.2624 & 0.3175 & 0.3355 & 0.3503 & 0.3168 & 0.3687 & 0.3388 \\
\hline & 25 & 0.3410 & 0.4586 & 0.4783 & 0.4864 & 0.4541 & 0.4965 & 0.4558 \\
\hline \multirow[t]{3}{*}{ Large $m_{i}$} & 25 & 0.2244 & 0.2845 & 0.3048 & 0.3069 & 0.2887 & 0.3124 & 0.2919 \\
\hline & 50 & 0.3584 & 0.4512 & 0.4620 & 0.4604 & 0.4564 & 0.4722 & 0.4468 \\
\hline & 100 & 0.5423 & 0.6580 & 0.6637 & 0.6643 & 0.6563 & 0.6630 & 0.6430 \\
\hline
\end{tabular}

TABLE 10

Power of CMM and Augmented tests: high correlation. Rows 1-3 contain results for cluster size $m_{i}=(20,30)$. Rows $4-6$ show results for $m_{i}=(4,6,8)$. (*) indicates model selection on precision matrix-transformed covariates and outcomes. Adaptive regression model selection: $A I C, B I C$ by n (BICn), BIC by $M,(B I C m)$, Adaptive LASSO (A. L.). Prespecified models: Correct (Corr.), Incorrect (Inco.). 'Unadj.' denotes the test statistic that does not incorporate baseline covariates.

Conditional Mean Model

\begin{tabular}{llllllllllll}
\hline$n_{a}$ & Unadj. & AIC & AIC $^{*}$ & BICn & BICn $^{*}$ & BICm & BICm* $^{*}$ & A. L. & A. L.* & Corr. & Inco. \\
\hline 10 & 0.252 & 0.524 & 0.482 & 0.527 & 0.477 & 0.519 & 0.467 & 0.503 & 0.487 & 0.409 & 0.356 \\
15 & 0.297 & 0.511 & 0.486 & 0.515 & 0.485 & 0.514 & 0.479 & 0.498 & 0.491 & 0.449 & 0.412 \\
25 & 0.350 & 0.544 & 0.532 & 0.547 & 0.531 & 0.549 & 0.526 & 0.537 & 0.527 & 0.504 & 0.477 \\
\hline 25 & 0.308 & 0.487 & 0.470 & 0.477 & 0.462 & 0.468 & 0.455 & 0.459 & 0.448 & 0.431 & 0.395 \\
50 & 0.466 & 0.611 & 0.606 & 0.605 & 0.603 & 0.604 & 0.603 & 0.600 & 0.599 & 0.590 & 0.558 \\
100 & 0.663 & 0.768 & 0.769 & 0.771 & 0.766 & 0.770 & 0.766 & 0.769 & 0.761 & 0.765 & 0.742 \\
\hline
\end{tabular}

Augmented

\begin{tabular}{llllllllllll}
\hline$n_{a}$ & Unadj. & AIC & AIC* & BICn & BICn* $^{*}$ & BICm & BICm* & A. L. & A. L. ${ }^{*}$ & Corr. & Inco. \\
\hline 10 & 0.252 & 0.630 & 0.547 & 0.527 & 0.459 & 0.493 & 0.434 & 0.607 & 0.566 & 0.449 & 0.401 \\
15 & 0.297 & 0.591 & 0.523 & 0.507 & 0.481 & 0.491 & 0.465 & 0.575 & 0.544 & 0.479 & 0.442 \\
25 & 0.350 & 0.583 & 0.551 & 0.539 & 0.533 & 0.532 & 0.523 & 0.578 & 0.557 & 0.524 & 0.488 \\
\hline 25 & 0.308 & 0.515 & 0.486 & 0.470 & 0.463 & 0.462 & 0.455 & 0.487 & 0.467 & 0.453 & 0.414 \\
50 & 0.466 & 0.623 & 0.608 & 0.602 & 0.603 & 0.602 & 0.601 & 0.613 & 0.605 & 0.598 & 0.563 \\
100 & 0.663 & 0.771 & 0.767 & 0.766 & 0.769 & 0.763 & 0.767 & 0.770 & 0.766 & 0.764 & 0.745 \\
\hline
\end{tabular}


TABLE 11

Power of Approximate Exact Tests: high correlation. Rows 1-3 contain results for cluster size $m_{i}=(20,30)$. Rows 4- 6 show results for $m_{i}=(4,6,8)$. (*) indicates model selection on precision matrix-transformed covariates and outcomes. Results based on Bickel's adjusted CDF are indicated by (Sm). Adaptive regression model selection: AIC, BIC by $n$ (BICn), BIC by M,(BICm), Adaptive LASSO (A. L.). Prespecified models: Correct (Corr.), Incorrect (Inco.). 'Unadj.' denotes the test statistic that does not incorporate baseline covariates.

Approximate Exact (Ind)

\begin{tabular}{llllllllllll}
\hline$n_{a}$ & Unadj. & AIC & AIC* & BICn & BICn* $^{*}$ & BICm & BICm* $^{*}$ & A. L. & A. L. $*$ & Corr. & Inco. \\
\hline 10 & 0.140 & 0.170 & 0.200 & 0.181 & 0.217 & 0.211 & 0.248 & 0.181 & 0.191 & 0.278 & 0.232 \\
15 & 0.197 & 0.270 & 0.316 & 0.280 & 0.328 & 0.306 & 0.340 & 0.279 & 0.299 & 0.355 & 0.322 \\
25 & 0.268 & 0.411 & 0.438 & 0.414 & 0.453 & 0.421 & 0.458 & 0.412 & 0.422 & 0.463 & 0.430 \\
\hline 25 & 0.213 & 0.328 & 0.351 & 0.342 & 0.365 & 0.352 & 0.367 & 0.340 & 0.344 & 0.375 & 0.342 \\
50 & 0.355 & 0.532 & 0.545 & 0.541 & 0.554 & 0.547 & 0.557 & 0.536 & 0.542 & 0.554 & 0.522 \\
100 & 0.557 & 0.733 & 0.744 & 0.740 & 0.749 & 0.743 & 0.749 & 0.734 & 0.736 & 0.744 & 0.717 \\
\hline
\end{tabular}

Approximate Exact (Ind-Sm)

\begin{tabular}{llllllllllll}
\hline$n_{a}$ & Unadj. & AIC & AIC $^{*}$ & BICn & BICn* $^{*}$ & BICm & BICm* $^{*}$ & A. L. & A. L. & Corr. & Inco. \\
\hline 10 & 0.142 & 0.173 & 0.205 & 0.185 & 0.223 & 0.216 & 0.252 & 0.185 & 0.194 & 0.284 & 0.235 \\
15 & 0.197 & 0.274 & 0.320 & 0.284 & 0.333 & 0.310 & 0.344 & 0.281 & 0.303 & 0.359 & 0.324 \\
25 & 0.270 & 0.413 & 0.441 & 0.417 & 0.454 & 0.423 & 0.460 & 0.415 & 0.425 & 0.466 & 0.432 \\
\hline 25 & 0.215 & 0.332 & 0.354 & 0.345 & 0.369 & 0.356 & 0.371 & 0.344 & 0.349 & 0.379 & 0.344 \\
50 & 0.357 & 0.535 & 0.549 & 0.546 & 0.558 & 0.550 & 0.561 & 0.540 & 0.548 & 0.558 & 0.525 \\
100 & 0.559 & 0.734 & 0.746 & 0.740 & 0.751 & 0.744 & 0.751 & 0.736 & 0.738 & 0.746 & 0.719 \\
\hline
\end{tabular}

Approximate Exact (Exch)

\begin{tabular}{llllllllllll}
\hline$n_{a}$ & Unadj. & AIC & AIC $^{*}$ & BICn & BICn* $^{*}$ & BICm & BICm* $^{*}$ & A. L. & A. L. $*$ & Corr. & Inco. \\
\hline 10 & 0.174 & 0.172 & 0.198 & 0.183 & 0.215 & 0.212 & 0.247 & 0.181 & 0.191 & 0.281 & 0.234 \\
15 & 0.239 & 0.274 & 0.320 & 0.287 & 0.333 & 0.311 & 0.345 & 0.283 & 0.300 & 0.359 & 0.322 \\
25 & 0.321 & 0.413 & 0.443 & 0.416 & 0.453 & 0.423 & 0.458 & 0.413 & 0.427 & 0.466 & 0.430 \\
\hline 25 & 0.266 & 0.334 & 0.356 & 0.348 & 0.371 & 0.360 & 0.374 & 0.341 & 0.350 & 0.380 & 0.346 \\
50 & 0.442 & 0.538 & 0.553 & 0.550 & 0.562 & 0.556 & 0.563 & 0.546 & 0.548 & 0.561 & 0.526 \\
100 & 0.649 & 0.740 & 0.748 & 0.748 & 0.751 & 0.754 & 0.752 & 0.742 & 0.742 & 0.753 & 0.732 \\
\hline
\end{tabular}

Approximate Exact (Exch-Sm)

\begin{tabular}{llllllllllll}
\hline$n_{a}$ & Unadj. & AIC & AIC $^{*}$ & BICn & BICn $^{*}$ & BICm & BICm* $^{*}$ & A. L. & A. L. $*$ & Corr. & Inco. \\
\hline 10 & 0.176 & 0.177 & 0.201 & 0.187 & 0.219 & 0.215 & 0.252 & 0.184 & 0.193 & 0.284 & 0.237 \\
15 & 0.240 & 0.278 & 0.323 & 0.291 & 0.337 & 0.313 & 0.348 & 0.286 & 0.304 & 0.363 & 0.325 \\
25 & 0.323 & 0.415 & 0.445 & 0.419 & 0.456 & 0.426 & 0.459 & 0.415 & 0.430 & 0.467 & 0.431 \\
\hline 25 & 0.268 & 0.339 & 0.360 & 0.353 & 0.374 & 0.365 & 0.377 & 0.346 & 0.354 & 0.385 & 0.349 \\
50 & 0.443 & 0.542 & 0.558 & 0.554 & 0.565 & 0.559 & 0.566 & 0.550 & 0.552 & 0.565 & 0.528 \\
100 & 0.650 & 0.743 & 0.751 & 0.750 & 0.752 & 0.755 & 0.754 & 0.744 & 0.746 & 0.755 & 0.733 \\
\hline
\end{tabular}


TABLE 12

Power of Exact Tests: high correlation. Rows 1-3 contain results for cluster size $m_{i}=(20,30)$. Rows 4-6 show results for $m_{i}=(4,6,8)$. Adaptive regression model selection: AIC, BIC by n (BICn), BIC by M,(BICm), Adaptive LASSO (A. L.).

Prespecified models: Correct (Corr.), Incorrect (Inco.). 'Unadj.' denotes the test statistic that does not incorporate baseline covariates.

Exact (Ind)

\begin{tabular}{llllllllllll}
\hline$n_{a}$ & Unadj. & AIC & AIC $^{*}$ & BICn & BICn* $^{*}$ & BICm & BICm $^{*}$ & A. L. & A. L. $^{*}$ & Corr. & Inco. \\
\hline 10 & 0.175 & 0.188 & 0.231 & 0.200 & 0.250 & 0.234 & 0.283 & 0.199 & 0.215 & 0.330 & 0.275 \\
15 & 0.222 & 0.294 & 0.347 & 0.304 & 0.367 & 0.331 & 0.380 & 0.302 & 0.330 & 0.410 & 0.366 \\
25 & 0.295 & 0.436 & 0.472 & 0.442 & 0.483 & 0.450 & 0.487 & 0.437 & 0.451 & 0.505 & 0.463 \\
\hline 25 & 0.231 & 0.351 & 0.379 & 0.365 & 0.392 & 0.378 & 0.398 & 0.367 & 0.374 & 0.409 & 0.370 \\
50 & 0.369 & 0.552 & 0.571 & 0.566 & 0.579 & 0.569 & 0.578 & 0.556 & 0.565 & 0.583 & 0.546 \\
100 & 0.571 & 0.748 & 0.758 & 0.754 & 0.762 & 0.759 & 0.761 & 0.752 & 0.752 & 0.761 & 0.732 \\
\hline
\end{tabular}

Exact (Exch)

\begin{tabular}{llllllllllll}
\hline$n_{a}$ & Unadj. & AIC & AIC* & BICn & BICn* $^{*}$ & BICm & BICm* & A. L. & A. L.* & Corr. & Inco. \\
\hline 10 & 0.217 & 0.189 & 0.229 & 0.201 & 0.250 & 0.234 & 0.284 & 0.199 & 0.215 & 0.330 & 0.277 \\
15 & 0.274 & 0.302 & 0.355 & 0.310 & 0.372 & 0.334 & 0.385 & 0.305 & 0.329 & 0.412 & 0.369 \\
25 & 0.343 & 0.438 & 0.474 & 0.442 & 0.483 & 0.450 & 0.490 & 0.439 & 0.457 & 0.503 & 0.467 \\
\hline 25 & 0.291 & 0.356 & 0.384 & 0.373 & 0.398 & 0.386 & 0.399 & 0.370 & 0.380 & 0.415 & 0.377 \\
50 & 0.461 & 0.558 & 0.574 & 0.572 & 0.585 & 0.577 & 0.587 & 0.564 & 0.572 & 0.589 & 0.553 \\
100 & 0.661 & 0.755 & 0.762 & 0.764 & 0.768 & 0.769 & 0.768 & 0.758 & 0.758 & 0.770 & 0.749 \\
\hline
\end{tabular}

